\title{
Quantum trajectories in spin-exchange collisions reveal the nature of spin-noise correlations in multispecies alkali-metal vapors
}

\author{
K. Mouloudakis, ${ }^{1}$ M. Loulakis, ${ }^{2,3}$ and I. K. Kominis $\oplus^{1,4, *}$ \\ ${ }^{1}$ Department of Physics, University of Crete, Heraklion 71003, Greece \\ ${ }^{2}$ School of Applied Mathematical and Physical Sciences, National Technical University of Athens, 15780 Athens, Greece \\ ${ }^{3}$ Institute of Applied and Computational Mathematics, FORTH, 70013 Heraklion, Greece \\ ${ }^{4}$ Institute of Theoretical and Computational Physics, University of Crete, Heraklion 70013, Greece
}

(Received 13 August 2019; published 10 October 2019)

\begin{abstract}
Spin-exchange collisions in alkali-metal vapors underlie several fundamental and applied investigations such as nuclear structure studies and tests of fundamental symmetries, ultrasensitive atomic magnetometers, magnetic resonance, and biomagnetic imaging. Spin-exchange collisions cause a loss of spin coherence and concomitantly produce spin noise, both phenomena being central to quantum metrology. We develop here the quantum-trajectory picture of spin-exchange collisions, consistent with their long-standing ensemble description using density matrices. We then use quantum trajectories to reveal the nature of spin-noise correlations that spontaneously build up in multispecies atomic vapors, frequently utilized in the most sensitive spin measurements.
\end{abstract}

DOI: 10.1103/PhysRevResearch.1.033017

\section{INTRODUCTION}

Atomic spin-exchange collisions are fundamental for a broad range of exploration, from nuclear physics and astrophysics to atomic spectroscopy, quantum metrology, and medical imaging. Spin-exchange collisions have been studied in the context of hyperfine transitions in hydrogen masers [1] and radio emission of interstellar hydrogen [2]. Spinexchange collisions in alkali-metal vapors have been central in producing nonequilibrium magnetic substate populations by optical pumping [3]. Spin-exchange optical pumping [4,5] has led to hyperpolarized noble gases used in medical imaging [6], spectroscopy [7], and numerous studies of nucleon spin structure [8]. Furthermore, the intricate physics of spinexchange collisions [9] has spurred the development of ultrasensitive magnetometers [10], allowing new precision tests of fundamental symmetries [11,12] and novel biomagnetic imaging applications $[13,14]$. Spin-exchange collisions cause spin coherence relaxation, and since relaxation and fluctuations are intimately connected, spin-exchange collisions are also underpinning spin noise spectroscopy [15-22].

So far, however, the understanding of spin-exchange collisions has been based on ensemble descriptions with density matrix master equations, which capture neither the physics at the single-atom level nor the spontaneous fluctuations of the collective spin or any phenomena stemming therefrom. We here use quantum measurement theory to shed light on the quantum foundations of spin-exchange collisions. We

\footnotetext{
*ikominis@physics.uoc.gr

Published by the American Physical Society under the terms of the Creative Commons Attribution 4.0 International license. Further distribution of this work must maintain attribution to the author(s) and the published article's title, journal citation, and DOI.
}

develop the quantum-trajectory picture of spin-exchange collisions [23] and demonstrate the consistency of our unraveling with the well-established density matrix master equation [24]. Quantum trajectories can be used to understand, at the single-atom level, alkali-metal-alkali-metal or even alkalimetal-noble-gas collisions [25]. We demonstrate how quantum trajectories can seamlessly produce spin noise from first principles. Moving to dual-species vapors, we use quantum trajectories to reveal the nature of spin-noise correlations that spontaneously build up $[26,27]$. As a by-product of the above, we present an approach to the stochastic terms augmenting the density matrix master equation describing spin-exchange collisions.

The structure of this article is the following. In Sec. II we briefly reiterate the long-standing ensemble description of spin-exchange collisions in hot atomic vapors, which uses a density matrix master equation. In Sec. III we introduce the quantum-trajectory picture of spin-exchange collisions and demonstrate its consistency with the ensemble description in physical scenarios involving large nonequilibrium spin polarizations but not fluctuations, i.e., in scenarios tractable with the master equation. We then show how quantum trajectories can seamlessly produce spin noise, which cannot be accounted for by the spin-exchange master equation. In Sec. IV we move to dual-species vapors and establish the consistency of the quantum-trajectory approach with the coupled master equations of the ensemble approach, again in physical scenarios involving large nonequilibrium spin polarizations but not fluctuations. In Sec. V we use quantum trajectories to address an open problem in spin-noise spectroscopy, namely, the spin-noise correlations that spontaneously build up in dual-species vapors. Finally, we augment the master equations describing spin-exchange collisions with physically realistic noise terms, rendering the description of spin noise possible at the ensemble level. The stochastic master equations 
provide an independent verification of the nature of spin-noise correlations.

\section{SPIN-EXCHANGE COLLISIONS: ENSEMBLE DESCRIPTION}

Spin-exchange (SE) collisions between two atoms A and $\mathrm{B}$, of the same or different species, result from the different interaction potentials $V_{S}$ and $V_{T}$ for the singlet and triplet total spin of the colliding partners, respectively. If $\mathbf{s}_{a}$ and $\mathbf{s}_{b}$ are the electron spins of the colliding atoms, the singlet and triplet projectors are $\mathcal{P}_{S}=\frac{1}{4} \mathbb{1}-\mathbf{s}_{a} \cdot \mathbf{s}_{b}$ and $\mathcal{P}_{T}=\frac{3}{4} \mathbb{1}+$ $\mathbf{s}_{a} \cdot \mathbf{s}_{b}{ }^{1}$ Introducing the exchange operator $\mathcal{P}_{e}=\mathcal{P}_{T}-\mathcal{P}_{S}$, the SE interaction potential $V_{\mathrm{SE}}=\mathcal{P}_{S} V_{S}+\mathcal{P}_{T} V_{T}$ is written as a sum of a spin-independent and a spin-dependent term $V_{\mathrm{SE}}=V_{0} \mathbb{1}+V_{1} \mathcal{P}_{e}$. Only the latter is of interest for the unitary operator evolving the initial into the final spin state, $U=$ $\exp \left(-i \int d t V_{1} \mathcal{P}_{e}\right)^{2}$ Defining $\phi=\int d t V_{1}$ and noting that $\mathcal{P}_{e}^{2}=\mathbb{1}$, we get $U=\cos \phi \mathbb{1}-i \sin \phi \mathcal{P}_{e} .^{3}$

For a single-species vapor of atoms A with number density [A] the ensemble description of SE collisions follows either [29] by using $\mathcal{P}_{e}$ and considering the SE rate $1 / T_{\mathrm{SE}}=[\mathrm{A}] \bar{v} \sigma_{\mathrm{SE}}$ or by using $U$ [24] and identifying $\overline{\sin ^{2} \phi} / T$ with $1 / T_{\mathrm{SE}}$, where $T$ the time between collisions and $\overline{\sin ^{2} \phi}$ the collisional average of $\sin ^{2} \phi$. Here $\sigma_{\mathrm{SE}}$ is the SE cross section and $\bar{v}$ the mean relative velocity of the colliding atoms. Neglecting the SE frequency shift [24], both approaches result in the same master equation. For following use, we here briefly reiterate the first approach.

Let two atoms A and B collide, initially assuming they are of different species, with their (uncorrelated) precollision states being $\rho_{a}$ and $\rho_{b}$. Hence the combined two-atom initial state is $\rho_{a} \otimes \rho_{b}$. The state of atom A after the collision is $\rho_{a b}^{e}=\operatorname{Tr}_{B}\left\{\mathcal{P}_{e} \rho_{a} \otimes \rho_{b} \mathcal{P}_{e}\right\}$,

$$
\begin{aligned}
\rho_{a b}^{e}= & \frac{1}{4} \rho_{a}+\mathbf{s}_{a} \cdot \rho_{a} \mathbf{s}_{a}+\left(\rho_{a} \mathbf{s}_{a}+\mathbf{s}_{a} \rho_{a}\right) \cdot\left\langle\mathbf{s}_{b}\right\rangle \\
& -2 i\left\langle\mathbf{s}_{b}\right\rangle \cdot\left(\mathbf{s}_{a} \times \rho_{a} \mathbf{s}_{a}\right) .
\end{aligned}
$$

For treating a single-species vapor, one substitutes $b \rightarrow a$ and arrives at the master equation describing both Hamiltonian evolution and SE collisions

$$
d \rho_{a} / d t=-i\left[\mathcal{H}_{a}, \rho_{a}\right]-\left(\rho_{a}-\rho_{a a}^{e}\right) / T_{\mathrm{SE}},
$$

where $\mathcal{H}_{a}=\omega s_{a z}+A \mathbf{s}_{a} \cdot \mathbf{I}_{a}$ is the alkali-metal ground-state Hamiltonian in the presence of a $z$-axis magnetic field $\omega$ and $A$ is the hyperfine coupling with the nuclear spin $\mathbf{I}_{a}$. The second term in Eq. (2) is responsible for transverse spin relaxation [30]. The above are well-known results, comprehensively presented in Ref. [24].

\footnotetext{
${ }^{1}$ We use $\mathbb{1}$ to denote the unit matrix of dimension that follows from the context, e.g., for two-body operators like $\mathcal{P}_{e}$ the unit matrix refers to the combined two-atom Hilbert space of dimension $2\left(2 I_{A}+1\right) 2\left(2 I_{B}+1\right)$.

${ }^{2}$ The time dependence of $V_{1}$ is implicit in its dependence on the internuclear distance, which changes with time along the collision trajectory.

${ }^{3}$ In the quantum information literature $\mathcal{P}_{e}$ and $U$ are more widely known as the SWAP and partial-SWAP operators, respectively (see, e.g., [28]).
}
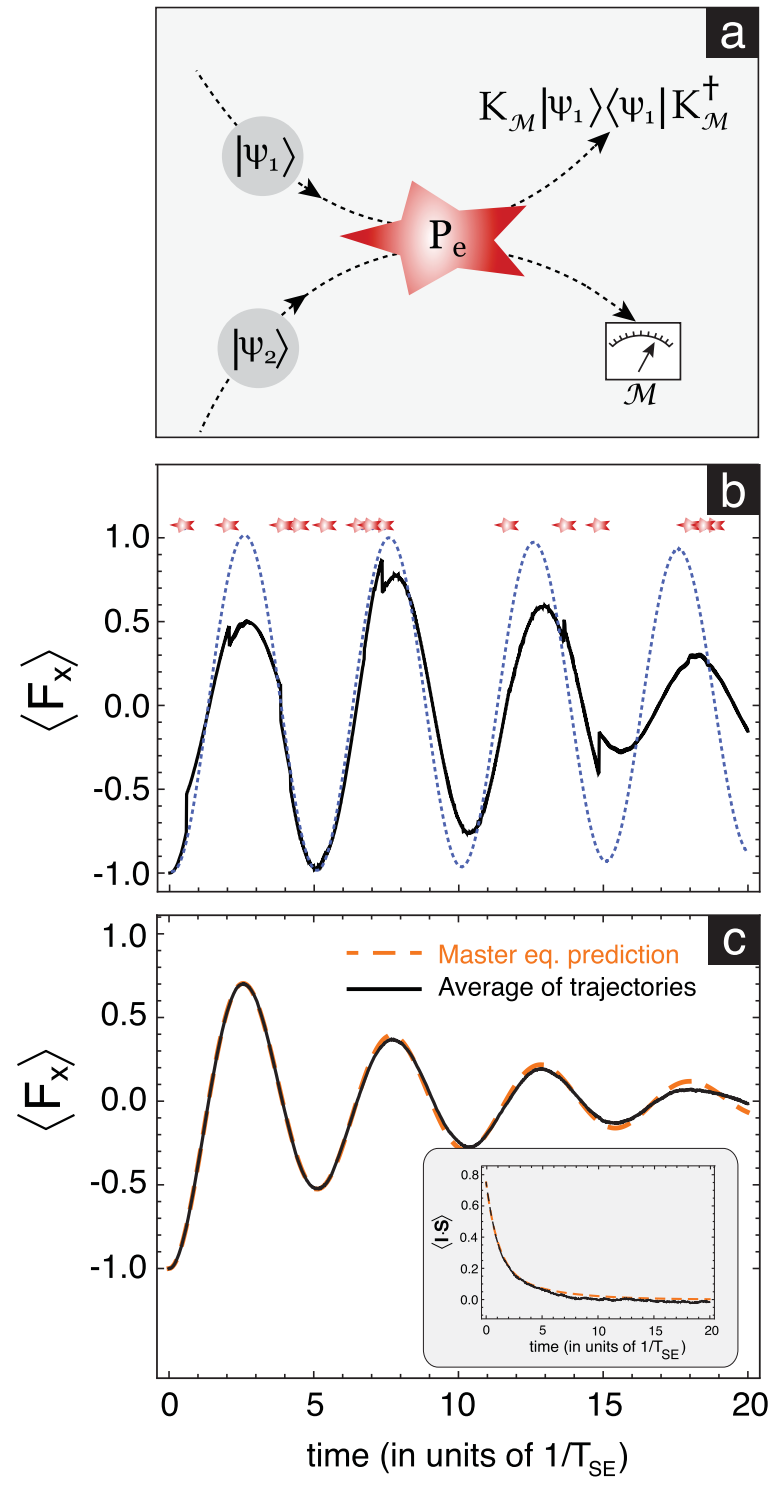

FIG. 1. (a) Quantum measurement picture of a binary spinexchange collision. (b) Single-atom trajectory expectation value of $\left\langle F_{x}\right\rangle$. Red stars depict the random occurrences of SE collisions. The chosen parameters were $I=3 / 2$ and in units of $1 / T_{\mathrm{SE}}, \omega=5$ and $A=100$, while time from $t=0$ to $t=20$ was split into $6 M$ steps. The initial state was the $|2-1\rangle$ eigenstate of $F_{x}$. The blue dotted line is just the Hamiltonian evolution. (c) Average (black solid line) of 4000 trajectories like in (b), compared with the predictions of Eq. (2) (orange dashed line). The inset is similar but for the observable $\langle\mathbf{I} \cdot \mathbf{s}\rangle$.

\section{SPIN-EXCHANGE COLLISIONS: QUANTUM-TRAJECTORY DESCRIPTION}

We will now develop the quantum-trajectory approach, pictured in Fig. 1(a), that is consistent with the above ensemble description. We start with two, initially uncorrelated, atoms 1 and 2 in the pure states $\left|\psi_{1}\right\rangle$ and $\left|\psi_{2}\right\rangle$. The combined two-atom precollision and postcollision states are $\left|\psi_{1}\right\rangle \otimes\left|\psi_{2}\right\rangle$ and $\mathcal{P}_{e}\left|\psi_{1}\right\rangle \otimes\left|\psi_{2}\right\rangle$, respectively. We now consider atom 1 as the system and atom 2 as the probe, from which we can extract information about the system. To do so, we perform a 
projective measurement on the probe. For this we here use the $|F M\rangle$ basis (zero-field eigenstates of $\mathbf{I} \cdot \mathbf{s}$ and $F_{z}$ ), but any other complete basis would do equally well. Atom 2 is projected in some state $|F M\rangle$ with probability, $p_{F M}$, given by the norm of the state $\Pi_{F M} \mathcal{P}_{e}\left|\psi_{1}\right\rangle \otimes\left|\psi_{2}\right\rangle$, where $\Pi_{F M}=$ $\mathbb{1} \otimes|F M\rangle\langle F M|$. Defining the Kraus operator corresponding to the particular measurement outcome on atom 2 as $\mathcal{K}_{F M}=$ $\left\langle F M\left|\mathcal{P}_{e}\right| \psi_{2}\right\rangle$, we find that $p_{F M}=\left\langle\psi_{1}\left|\mathcal{K}_{F M}^{\dagger} \mathcal{K}_{F M}\right| \psi_{1}\right\rangle$. Concomitantly, atom 1 is projected to

$$
\left|\psi_{1}^{e}\right\rangle_{F M}=\frac{\mathcal{K}_{F M}\left|\psi_{1}\right\rangle}{\sqrt{p_{F M}}}
$$

The Kraus operators satisfy the completeness relation $\sum_{F M} \mathcal{K}_{F M}^{\dagger} \mathcal{K}_{F M}=\mathbb{1}$, which readily follows from the completeness of the $|F M\rangle$ basis states and the property $\mathcal{P}_{e}^{2}=\mathbb{1}^{4}$ Hence it is also $\sum_{F M} p_{F M}=1$, as it should be. Finally, the state $\left|\psi_{1}^{e}\right\rangle_{F M}$ is properly normalized.

For the numerical production of quantum trajectories, we consider $N$ atoms, each in any desired pure initial state. We split time into intervals $d t,{ }^{5}$ and in every $d t$ we evolve each atom unitarily with the Hamiltonian. Moreover, in each $d t$ there is a probability $d t / T_{\mathrm{SE}}$ that an atom suffers an $\mathrm{SE}$ collision. A random number drawn for each atom determines whether this probability is realized. For those atoms (system atom 1) undergoing an SE collision during $d t$, the collision partner (probe atom 2) is randomly chosen from the same list of $N$ atoms. Finally, given $p_{F M}$, we let another random number determine what the projective measurement outcome $|F M\rangle$ of atom 2 is. Then atom 1 is projected to $\left|\psi_{1}^{e}\right\rangle_{F M}$ given by Eq. (3).

Crucially, although the particular projection of the probe atom to a state $|F M\rangle$ determines the projection of the system atom to $\left|\psi_{1}^{e}\right\rangle_{F M}$, after the collision we leave atom 2 in its initial precollision state. This has imprinted a Markovian interpretation, i.e., all probe atoms instantly lose memory of the collisions taking place during $d t$ and reconstitute the ensemble precollision state. We here do not investigate whether the above picture is physically precise, but only care to lay out the single-atom physics behind the master equation (2), which has been impressively successful in accounting for a broad set of experimental data.

Having access to $N$ quantum trajectories, we can evaluate the time evolution of any observable $Q$ either for the particular $j$ th trajectory, as $\langle Q\rangle_{t}^{(j)}=\left\langle\psi_{j}(t)|Q| \psi_{j}(t)\right\rangle$, or for the trajectory average used to compare with the master equation, as $\langle Q\rangle_{t}=\frac{1}{N} \sum_{j=1}^{N}\langle Q\rangle_{t}^{(j)}$. In Fig. 1(b) we choose for $Q$ the transverse spin $F_{x}$ and show an example of a single trajectory depicting the discontinuities due to SE collisions. In Fig. 1(c) we show how the average of many such trajectories accounts

\footnotetext{
${ }^{4}$ Here the unit operator refers to the single-atom Hilbert space, because $\mathcal{K}_{F M}$ are single-atom operators.

${ }^{5}$ The value of $d t$ is chosen so that oscillating observables are numerically well represented. Since realistic values of $\omega$ and $A$ in the Hamiltonian $\mathcal{H}$ would require such a small value of $d t$ that the simulation would not run in realistic times, we here choose unrealistically small hyperfine couplings, which in any case do not affect the physical considerations herein.
}
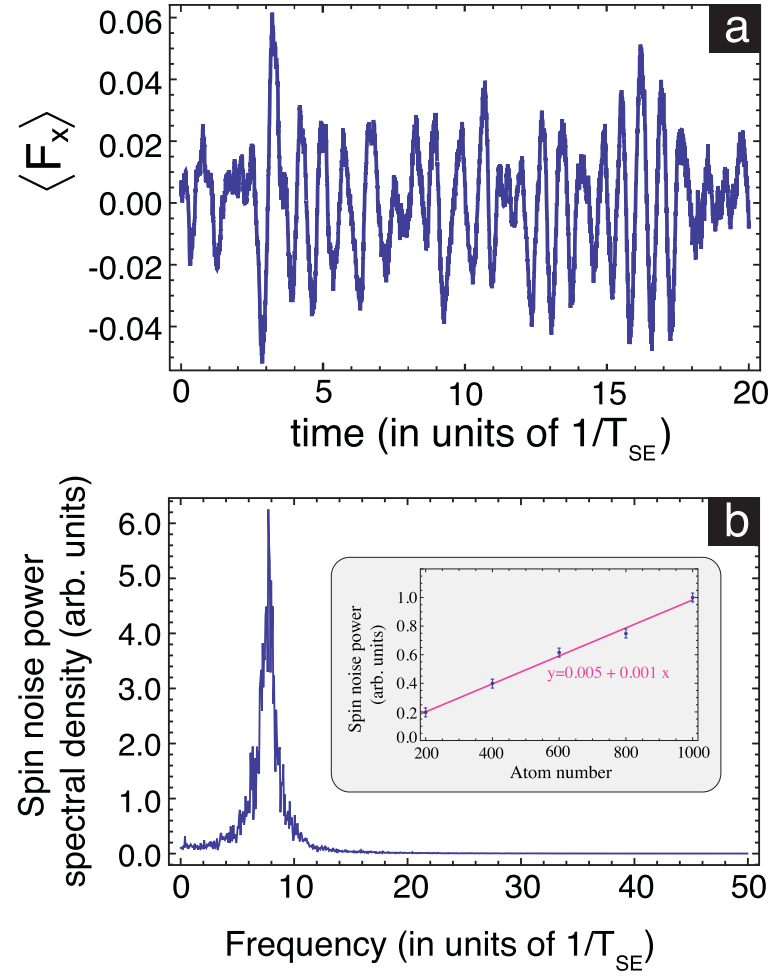

FIG. 2. (a) Spin noise produced by randomly choosing one of the eigenstates of $F_{x}$ as the initial state for each of 400 trajectories. Here $I=3 / 2, \omega=32$, and $A=100$. (b) Spin noise spectrum resulting from the average of 50 FFT power spectra derived from 50 time traces like (a). The inset shows the linear dependence of the total spin noise power of a single FFT power spectrum as a function of atom number. Error bars were estimated from two different FFT power spectra.

for spin relaxation at the ensemble level and compare the trajectory average with Eq. (2), both for $F_{x}$ and for a second observable I $\cdot \mathbf{s}$. The perfect agreement demonstrates the consistency of our quantum-trajectory approach.

We next show how the trajectory picture produces spin noise. We choose for each of the $N$ atoms a random initial state among all eigenstates of $F_{x}$. In Fig. 2(a) we plot the resulting fluctuations of $\left\langle F_{x}\right\rangle$ and in Fig. 2(b) a fast Fourier transform (FFT) spectrum that is typically observed in spinnoise spectroscopy. We note that the spin noise in Fig. 2(a) is not due to an imbalance of the initial states in a finite number of $N$ trajectories. Similar spin-noise traces can be produced even by exactly balancing the initial states so that at $t=0$ it is $\left\langle F_{x}\right\rangle=0$ and even by using as initial states eigenstates of $F_{z}$. Hence spin noise produced by SE collisions is a genuine quantum effect originating from the quantum randomness of the postcollision states.

\section{SPIN-EXCHANGE COLLISIONS IN DUAL-SPECIES VAPORS: ENSEMBLE VERSUS QUANTUM-TRAJECTORY APPROACH}

We now move to the case of a dual-species vapor of atoms $\mathrm{A}$ and $\mathrm{B}$ with relative abundances $\eta_{a}$ and $\eta_{b}\left(\eta_{a}+\right.$ $\left.\eta_{b}=1\right)$. This is treated similarly, using $N$ atoms with 
ground-state Hamiltonian $H_{a}$ and another $N$ atoms with ground-state Hamiltonian $H_{b}$. Now we need to introduce four kinds of collisions, i.e., A-A, A-B, B-A, and B-B collisions, with the respective relaxations rates $\gamma_{\alpha \beta}$ and SE collision probabilities $d P_{\alpha \beta}=\gamma_{\alpha \beta} d t$. The rates $\gamma_{\alpha \beta}$ are proportional to the mean relative $\alpha-\beta$ velocity and to the abundance $\eta_{\beta}$, i.e., $\gamma_{\alpha \beta} \propto \bar{v}_{\alpha \beta} \eta_{\beta}$, since the SE relaxation rate of the first collision partner is proportional to the atom number density of the second collision partner. Given $d P_{\alpha \beta}$, we let random numbers determine whether each of the $2 N$ atoms will perform a selfand/or a cross-exchange collision and we again randomly choose the collision partner.

Extending Eq. (2) to a dual-species vapor, we arrive at the two coupled master equations (derived in Ref. [24])

$$
\begin{aligned}
\frac{d \rho_{a}}{d t} & =-i\left[\mathcal{H}_{a}, \rho_{a}\right]-\gamma_{a a}\left(\rho_{a}-\rho_{a a}^{e}\right)-\gamma_{a b}\left(\rho_{a}-\rho_{a b}^{e}\right), \\
\frac{d \rho_{b}}{d t} & =-i\left[\mathcal{H}_{b}, \rho_{b}\right]-\gamma_{b a}\left(\rho_{b}-\rho_{b a}^{e}\right)-\gamma_{b b}\left(\rho_{b}-\rho_{b b}^{e}\right),
\end{aligned}
$$

where $\rho_{b a}^{e}$ and $\rho_{b b}^{e}$ follow from $\rho_{a b}^{e}$ in Eq. (1) by exchanging $a \leftrightarrow b$ and by substituting $a \rightarrow b$, respectively. We provide two examples of the consistency between Eqs. (4) and (5) and the trajectory average. In Fig. 3(a) we demonstrate the transfer of spin polarization from type-B atoms to type-A atoms. This is described by the simple equation $d\left\langle s_{z}\right\rangle_{a} / d t=$ $\left(\left\langle s_{z}\right\rangle_{b}-\left\langle s_{z}\right\rangle_{a}\right) / T_{\mathrm{SE}}[31]$ and can be experimentally realized by optically pumping with circularly polarized light atoms B and measuring the spin polarization building up in atoms A. To translate these dynamics into the trajectory picture we use a $+1 / 2$ eigenstate of $s_{z}$ as the initial state of atoms B and an equal mixture of eigenstates of $s_{z}$ for atoms A. Atoms B are always kept in their initial state, and we consider only A-B collisions. Next we consider coherent dynamics, by setting the initial spin polarization of atoms B transversely to the magnetic field and by again having atoms A unpolarized. We now observe simultaneously (i) the precession, (ii) the cross transfer, and (iii) the decay of the transverse polarizations, as shown in the inset of Fig. 3(a). Perfect agreement is again observed for both cases.

\section{SPIN-NOISE CORRELATIONS IN DUAL-SPECIES VAPORS}

Having established the consistency of our trajectory approach with the coupled dynamics of Eqs. (4) and (5), we now move to analyze spin noise correlations that spontaneously build up in coupled vapors [26,27]. Like in Fig. 2(a), we generate spin-noise time traces $\left\langle s_{x}\right\rangle_{a}^{(j)}$ and $\left\langle s_{x}\right\rangle_{b}^{(j)}$ in a dual-species vapor and do so for various magnetic fields $\omega$. The index $j$ is in the range $j=1, \ldots, j_{\max }$, where $j_{\max } d t$ is the total time interval considered. In Fig. 3(b) we show the correlation coefficient $\psi_{a b}^{\omega}$ for each magnetic field value $\omega$, where

$$
\psi_{a b}^{\omega}=\frac{\sum_{j}\left(\left\langle s_{x}\right\rangle_{a}^{(j)}-\overline{\left\langle s_{x}\right\rangle_{a}}\right)\left(\left\langle s_{x}\right\rangle_{b}^{(j)}-\overline{\left\langle s_{x}\right\rangle_{b}}\right)}{\sqrt{\sum_{j}\left(\left\langle s_{x}\right\rangle_{a}^{(j)}-\overline{\left\langle s_{x}\right\rangle_{a}}\right)^{2} \sum_{j}\left(\left\langle s_{x}\right\rangle_{b}^{(j)}-\overline{\left\langle s_{x}\right\rangle_{b}}\right)^{2}}} .
$$

At low $\omega$ we observe positive correlations, which then tend to zero as $\omega$ increases. This effect was measured in Ref. [26] and was theoretically derived from the coupled Bloch equations augmented with noise generating terms. Here the
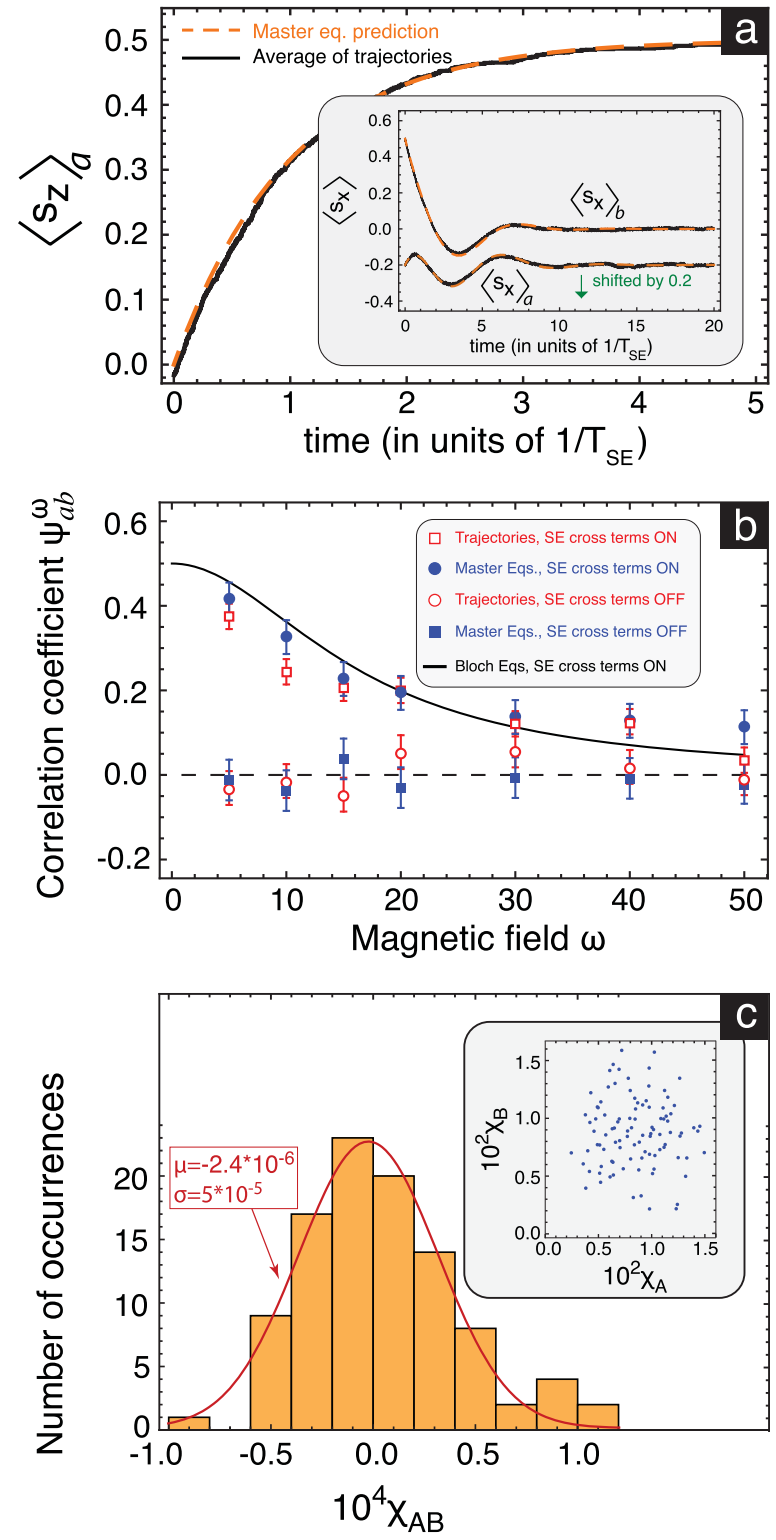

FIG. 3. (a) Spin transfer from 1000 atoms B $(I=5 / 2)$, always kept in a $+1 / 2$ eigenstate of $s_{z}$, to 1000 atoms A $(I=3 / 2)$, initially unpolarized. The trajectory average (black solid line) is compared with Eq. (4), keeping only the cross-exchange term (dashed orange line). The inset shows a similar comparison with the B atoms initially in the $|33\rangle$ eigenstate of $F_{x}$. For both vapors we took $A=50$ and $\omega=$ 5. The trace for $\left\langle s_{x}\right\rangle_{a}$ is shifted downward for clarity. (b) Spin-noise correlation coefficient $\psi_{a b}^{\omega}$ for 400 atoms of type A and 400 atoms of type B. Time from $t=0$ to $t=20$ was split into $2 M$ steps. Initially, each atom is in a random eigenstate of $F_{x}$. Every point is the mean of ten runs, and the error bar is the standard error of the mean. Shown are the trajectory average and the stochastic coupled master equation prediction, with and without cross exchange. The solid line is the prediction of the theory developed in Ref. [26]. (c) Distribution of $\chi_{a b}, \chi_{a}$, and $\chi_{b}$ for 100 spin noise runs with randomized $5 \leqslant \omega \leqslant 50$ and $0.1 \leqslant \gamma_{\alpha \beta} \leqslant 1.5$.

positive correlation effect is demonstrated with a firstprinciples quantum-trajectory analysis without any assumption. The coupled Bloch equations prediction is also shown in Fig. 3(b) for completeness. 
Importantly, we here move beyond the Bloch equations and further support the positive correlation effect using Eqs. (4) and (5). To do so, these equations need to be augmented with noise terms, which are operators acting in the relevant Hilbert space, whereas in the Bloch equations [26] the noise terms were just $c$-numbers. The first-principles derivation of these noise operators is not addressed here. Here we make an ad hoc but physically realistic assumption about their form and show that the stochastic master equation prediction for $\psi_{a b}^{\omega}$ agrees with the trajectory average.

Explicitly, since spin noise produces spin polarization of order $1 / \sqrt{N} \ll 1$, we assume that the atom's density matrix describing spin noise produced by SE collisions follows a spin-temperature distribution [5] with a fluctuating albeit high temperature, i.e., $\rho=e^{-\lambda F_{x}} / \operatorname{Tr}\left\{e^{-\lambda F_{x}}\right\}$, with $\lambda \ll 1$. Expanding $\rho$ around $\lambda=0$, we find for the differential change $\delta \rho \propto F_{x}$. Hence we set for the stochastic terms added to (4) and (5) $\delta_{t} \rho_{a}=\sqrt{\frac{\gamma_{a}}{N_{a}}} F_{x} d \xi_{t}^{a}$ and $\delta_{t} \rho_{b}=\sqrt{\frac{\gamma_{b}}{N_{b}}} F_{x} d \xi_{t}^{b},{ }^{6}$ respectively, where $\gamma_{a}=\gamma_{a a}+\gamma_{a b}$ and $\gamma_{b}=\gamma_{b a}+\gamma_{b b},{ }^{7}$ while $d \xi_{t}{ }^{a}$ and $d \xi_{t}^{b}$ are real and independent Wiener processes with zero mean and variance $d t$, i.e., $\left\langle\left\langle d \xi_{t}^{a}\right\rangle\right\rangle=\left\langle\left\langle d \xi_{t}^{b}\right\rangle\right\rangle=0$ and $\left\langle\left\langle d \xi_{t}^{\alpha} d \xi_{t^{\prime}}^{\beta}\right\rangle\right\rangle=d t \delta_{\alpha \beta} \delta\left(t-t^{\prime}\right)$, with $\alpha, \beta=a, b$. The prediction of the stochastic master equations is shown in Fig. 3(b) to reproduce the trajectory average.

As a systematic check, we turn off the cross-exchange process in the generation of trajectories, i.e., we do not perform A-B and B-A collisions. Similarly, we turn off the crossexchange coupling terms in the stochastic coupled master equations. As shown in Fig. 3(b), $\psi_{a b}^{\omega}$ is consistent with zero for both cases.

\footnotetext{
${ }^{6} \mathrm{We}$ note that the operator $F_{x}$ is a different matrix in the two noise operators, since they refer to different atom species, i.e., with different nuclear spin, and hence different Hilbert spaces.

${ }^{7}$ The particular numerical value in front of the operator $F_{x}$ in the noise terms is inconsequential for this work, since it drops out of the A-B correlation coefficient.
}

Finally, the fact that the noise terms $d \xi_{t}^{a}$ and $d \xi_{t}^{b}$ should be independent can be further supported by the quantum trajectories, from which we calculate $\chi_{a a}, \chi_{b b}$, and $\chi_{a b}$, where $\chi_{\alpha \beta}=$ $\sum_{j}\left(\left\langle s_{x}\right\rangle_{\alpha}^{(j+1)}-\left\langle s_{x}\right\rangle_{\alpha}^{(j)}\right)\left(\left\langle s_{x}\right\rangle_{\beta}^{(j+1)}-\left\langle s_{x}\right\rangle_{\beta}^{(j)}\right)$, with $\alpha, \beta=a, b$. We do this for 100 spin noise runs, with randomized rates $\gamma_{\alpha \beta}$ and magnetic field $\omega$. In Fig. 3(c) it can be seen that $\chi_{a b}$ is about three orders of magnitude less than $\chi_{a}$ and $\chi_{b}$. Yet, a standard result [32] on the quadratic variation of an Ornstein-Uhlenbeck diffusion process is that (as $d t \rightarrow$ 0) $\chi_{\alpha \beta} \propto\left\langle\left\langle d \xi_{t}^{\alpha} d \xi_{t}^{\beta}\right\rangle\right\rangle$. Thus, spin-noise correlations in dualspecies vapors are consistent with independent noise terms driving the master equations (4) and (5), the correlations being produced by the cross-couplings terms in (4) and (5) and not by any conspicuous choice of the noise terms.

\section{CONCLUSION}

We have developed a single-atom quantum-trajectory picture of spin-exchange collisions consistent with the density matrix ensemble description used so far. This picture is ideally suited to understand quantum fluctuations of all sorts of spin observables, the fluctuations being driven by the incessant atomic collisions and the resulting binary spin-exchange interaction. In our application, we demonstrated from first principles that spin-exchange collisions spontaneously produce positive spin-noise correlations in vapors containing two atomic species.

\section{ACKNOWLEDGMENTS}

K.M. acknowledges the cofinancing of this research by Greece and the European Union (European Social Fund) through the Operational Programme Human Resources Development, Education and Lifelong Learning in the context of the project "Strengthening Human Resources Research Potential via Doctorate Research" (Project No. MIS-5000432), implemented by the State Scholarships Foundation (I.K.Y.).
[1] R. L. Walsworth, I. F. Silvera, E. M. Mattison, and R. F. C. Vessot, Phys. Rev. Lett. 64, 2599 (1990).

[2] B. Zygelman, Astrophys. J. 622, 1356 (2005).

[3] W. Happer, Rev. Mod. Phys. 44, 169 (1972).

[4] T. G. Walker and W. Happer, Rev. Mod. Phys. 69, 629 (1997).

[5] S. Appelt, A. B.-A. Baranga, C. J. Erickson, M. V. Romalis, A. R. Young, and W. Happer, Phys. Rev. A 58, 1412 (1998).

[6] M. S. Albert, G. D. Cates, B. Driehuys, W. Happer, B. Saam, C. S. Springer, Jr., and A. Wishnia, Nature (London) 370, 199 (1994).

[7] G. Navon, Y. Q. Song, T. Room, S. Appelt, R. E. Taylor, and A. Pines, Science 271, 1848 (1996).

[8] A. Deur, S. J. Brodsky, and G. F. de Téramond, Rep. Prog. Phys. 82, 076201 (2019).

[9] W. Happer and A. C. Tam, Phys. Rev. A 16, 1877 (1977).

[10] D. Budker and M. V. Romalis, Nat. Phys. 3, 227 (2007).

[11] M. Smiciklas, J. M. Brown, L. W. Cheuk, S. J. Smullin, and M. V. Romalis, Phys. Rev. Lett. 107, 171604 (2011).
[12] M. S. Safronova, D. Budker, D. DeMille, D. F. J. Kimball, A. Derevianko, and C. W. Clark, Rev. Mod. Phys. 90, 025008 (2018).

[13] H. Xia, A. Ben-Amar Baranga, D. Hoffman, and M. V. Romalis, Appl. Phys. Lett. 89, 211104 (2006).

[14] T. Sander, J. Preusser, R. Mhaskar, J. Kitching, L. Trahms, and S. Knappe, Biomed. Opt. Express 3, 981 (2012).

[15] E. B. Aleksandrov and V. S. Zapasskii, Sov. Phys. JETP 54, 64 (1981).

[16] S. A. Crooker, D. G. Rickel, V. A. Balatsky, and D. L. Smith, Nature (London) 431, 49 (2004).

[17] M. Oestreich, M. Römer, R. J. Haug, and D. Hägele, Phys. Rev. Lett. 95, 216603 (2005).

[18] G. E. Katsoprinakis, A. T. Dellis, and I. K. Kominis, Phys. Rev. A 75, 042502 (2007).

[19] V. S. Zapasskii, A. Greilich, S. A. Crooker, Y. Li, G. G. Kozlov, D. R. Yakovlev, D. Reuter, A. D. Wieck, and M. Bayer, Phys. Rev. Lett. 110, 176601 (2013). 
[20] N. A. Sinitsyn and Y. V. Pershin, Rep. Prog. Phys. 79, 106501 (2016).

[21] V. G. Lucivero, R. Jiménez-Martínez, J. Kong, and M. W. Mitchell, Phys. Rev. A 93, 053802 (2016).

[22] J. Kong, R. Jiménez-Martínez, C. Troullinou, V. G. Lucivero, and M. W. Mitchell, arXiv:1804.07818.

[23] I. H. Deutsch and P. S. Jessen, Opt. Commun. 283, 681 (2010).

[24] W. Happer, Y.-Y. Jau, and T. G. Walker, Optically Pumped Atoms (Wiley-VCH, Weinheim, 2010).

[25] O. Katz, R. Shaham, and O. Firstenberg, arXiv:1905.12532.
[26] A. T. Dellis, M. Loulakis, and I. K. Kominis, Phys. Rev. A 90, 032705 (2014).

[27] D. Roy, L. Yang, S. A. Crooker, and N. A. Sinitsyn, Sci. Rep. 5, 9573 (2015).

[28] T. A. Brun, Am. J. Phys. 70, 719 (2002).

[29] F. Grossetête, J. Phys. (Paris) 25, 383 (1964); 29, 456 (1968).

[30] I. M. Savukov and M. V. Romalis, Phys. Rev. A 71, 023405 (2005).

[31] W. Happer, Hyperfine Interact. 38, 35 (1987).

[32] D. Revuz and M. Yor, Continuous Martingales and Brownian Motion (Springer Science + Business Media, New York, 2013). 